\title{
Influence of Multiwalled Carbon Nanotubes in Polytetrafluoroethylene on the Parameters of Electronic Structure and Absorption of UHF Radiation
}

\section{Iryna Galstian ( $\nabla$ stenforti@ukr.net )}

G.V. Kurdyumov Institute for Metal Physics N.A.S.U.

\section{Yevgen Tsapko}

G.V. Kurdyumov Institute for Metal Physics N.A.S.U.

\section{Olexiy Makarenko}

Taras Shevchenko National University of Kyiv
A.L. Yampolskiy
Taras Shevchenko National University of Kyiv

\section{Research Article}

Keywords: electronic structure, positron spectroscopy, optical properties of the composites, multiwalled carbon nanotubes

Posted Date: January 20th, 2022

DOI: https://doi.org/10.21203/rs.3.rs-1278942/v1

License: (c) (i) This work is licensed under a Creative Commons Attribution 4.0 International License.

Read Full License 


\section{Abstract}

Using the methods of angular correlation of annihilation radiation, attenuation of electromagnetic radiation in 1.5-2 $2 \mathrm{GHz}$ frequency range, and optical ellipsometry, it was shown that a $2 \%$ decrease in the probability of annihilation of positrons in pores of free volume leads to changes in the parameters of electronic structure of composites by $15-25 \%$ in composites of polytetrafluoroethylene (PTFE) + multiwalled carbon nanotubes (MWCNTs). Polytetrafluoroethylene is transparent to electromagnetic radiation, but after the addition of $10 \mathrm{wt} \%$ multiwalled carbon nanotubes, the composite demonstrates a more than 400 -fold decrease in the electromagnetic radiation intensity when the radiation passes through a specimen with a thickness of $\approx 2 \mathrm{~mm}$. It was found that the average radius of the free volume pores and the probability of annihilation of positrons in this area are determined by the electronic structure parameters of the polymer matrix only. The Fermi angle and the probability of positrons annihilation with free electrons are determined by the electronic structure parameters of MWCNTs only. Since the electronic structure of the cores and defects of the polymer matrix (at least outside the interphase) does not change, changes in these characteristics are mainly due to changes in the MWCNTs electronic structure. It was found that in a specimen with $10 \mathrm{wt} \%$ MWCNTs, the highest density of free electrons is observed, and the highest electron density is observed on defects. The average of free volume pore radius reaches its maximum value at $10 \mathrm{wt} \%$ MWCNTs in the composite.

\section{Introduction}

In (Tsapko E. A. (2020), Nishchenko M. M. (2011), Lihtorovich S. P. (2010)), the authors have shown that within the framework of positron spectroscopy (PS), it is possible to obtain such parameters of the electronic structure of multiwalled carbon nanotubes (MWCNTs) as the layer thickness, interlayer distance and effective pore radius of the free volume. The addition of nanoscale reinforcement (such as MWCNTs or graphene) to polymer matrices improves the structure, electrical, mechanical, and thermal properties of the polymer. The large surface area of the filler (MWCNT or graphene) leads to an increase in the filler-polymer interaction, which improves the transfer of load between the filler and the matrix, while interconnected contacts create electrical and thermal conductivity of typically non-conductive polymer materials, and increase at ultrahigh frequencies the radio absorption by several orders of magnitude (Lihtorovich S. P. (2010), Awad S. (2019)a, Xue G. (2016)).

In this work, within the framework of the methods of angular correlation of annihilation radiation (ACAR), attenuation of electromagnetic radiation, and optical ellipsometry, we show that in composites of polytetrafluoroethylene-multiwalled carbon nanotubes (PTFE-MWCNT), the probability of positron annihilation in pores of free volume decreases by $2 \%$ leads to changes in the parameters of the electronic structure of composites by $15-25 \%$, and PTFE, which is completely transparent to electromagnetic radiation, after the addition of $10 \mathrm{wt}$.\% MWCNT absorbs electromagnetic radiation at a frequency of 2 $\mathrm{GHz}$ by almost $100 \%$.

\section{Experimental}


The investigated samples of PTFE-MWCNT composites were obtained by the method described in (Poperenko L. V. (2007)). The structural characteristics of MWCNTs and PTFE-MWCNT composites were determined by the method of transmission microscopy (TEM, JEM-100CXII). The average diameter of the MWCNTs used in the composites was 10-20 nm, the specific surface, which was determined by the desorption of argon, was $200-400 \mathrm{~m}^{2} / \mathrm{g}$, and the bulk density varied in the range of $20-40 \mathrm{~g} / \mathrm{dm}^{3}$.

We investigated mechanically ground PTFE-MWCNT disks with a diameter of $20 \mathrm{~mm}$ and a thickness of $4 \mathrm{~mm}$ with concentrations of $0,5,10,15$, and $20 \mathrm{wt} . \%$ MWCNT. The measurements were carried out in the air at room temperature.

The measurements of the attenuation of electromagnetic radiation of the samples were carried out on an automatic attenuation meter $\mathrm{P} 2-52 / 3$ in the frequency range $1.5-2.0 \mathrm{GHz}$ according to the standard scheme for measuring microwave attenuation in four-pole networks using a copper standard (Lihtorovich S. P. (2010)).

The optical properties of the samples (the spectral dependences of the refractive indices $n$ and absorption $k$ ) were obtained using angular ellipsometry (Poperenko L. V. (2007)).

The ACAR spectra for the samples were obtained using a long-slit spectrometer with an angular resolution of $1.07 \mathrm{mrad}$.

To interpret the experimental data we used the classical analytical representation of the ACAR spectra as the sum of the parabolic part due to the annihilation of positrons with free electrons, two Gaussian parts due to the annihilation of positrons with core electrons of carbon and fluorine, in Stone-Welsh defects in MWCNTs and PTFE polymer chains, and a narrow Gaussian part (with variance from 0.2 to 1.2 mrad.), due to annihilation "on the catch" from the state of ortho-positronium (o-Ps) (Tsapko E. A. (2020), Yang M. K. (2008)):

$$
I^{\text {theor }}(\theta)=I_{P}\left[\frac{\theta_{F}^{2}-\theta^{2}}{2}+A_{B} \operatorname{Tln}\left(1+\exp \left\{-\frac{\theta_{F}^{2}-\theta^{2}}{2 A_{B} T}\right\}\right)\right]+\sum_{j=1}^{3} \frac{I_{G}^{j}}{\sigma_{j} \sqrt{2 \Pi}} \exp \left\{-\frac{\theta^{2}}{2 \sigma_{j}^{2}}\right\}(1)
$$

where $\theta$ is the deviation between the angle of expansion of annihilation $y$-quanta from $\pi, t^{\text {theor }}(\theta)$ is the analytical representation of the ACAR, $I_{P}, I_{G}^{j}$ are the amplitude coefficients (intensities) of the parabolic and Gaussian parts of the angular correlation of annihilation photons spectra, respectively, $\sigma_{j}$ is the dispersion of Gaussians, $\theta_{F}$ is the angle corresponding to the Fermi momentum, $A_{B}$ is the renormalized Boltzmann constant, $T$ is the sample temperature.

From the ACAR spectra, we obtain the Fermi momentum $P_{F}$ related to the angle $\theta_{F}$ by the ratio:

$$
P_{F}=m c \theta_{F}
$$


where $m$ is the mass of an electron, $c$ is the speed of light.

By integrating the parabolic part and the full spectrum of the ACAR over the angles, we obtain the

probability of annihilation of positrons with free electrons $P_{\theta_{F}}$ as the ratio of the area under the parabolic part to the total area under the ACAR.

The dispersion $\sigma_{j}$ of Gaussians, which determine the widths of the momentum distributions of electrons, are inversely proportional to the widths of their spatial distribution and are related to the distance $r_{m_{j}}$ from the center of the Wigner-Seitz cell, at which the overlap of the wave functions of the annihilating positron and electron is maximum, by the relation (Tsapko E. A. (2020)):

$$
r_{m_{j}}=\sqrt{\frac{3}{2}} \frac{\square}{m c \sigma_{j}}
$$

3

where $m$ is the mass of an electron, $c$ is the speed of light, $\hbar$ is Planck's constant.

The probabilities of annihilation of positrons with bound electrons $P_{r_{m_{j}}}$ are defined as the ratio of the areas under the corresponding components to the area under the full spectrum of the ACAR.

Through the dispersion of a narrow Gaussian $\mathbb{B}_{n}$ in the framework of the Tao model (Tao S. J. J (1972)), we can calculate the average radius $R$ of the free volume pores:

$\mathrm{R}=\backslash$ frac $\{0.74049\}\{\{\mathbb{X}\}\{\{\mathrm{n}\}\}-0.1656, \mathrm{~nm}$

4

The ACAR spectrum for MWCNTs consists of 3 components (2 Gaussians: the average one due to the annihilation of positrons with the carbon atoms core electrons, the broad one due to the annihilation of positrons in Stone-Welsh defects, and the parabolic contribution due to the annihilation of positrons with free electrons). While the ACAR spectrum for PTFE-MWCNT composites consists of 4 components ( 3 Gaussians: a wide one, caused by the annihilation of positrons in Stone-Welsh defects in MWCNTs and PTFE polymer chains, an average one caused by annihilation of positrons with carbon core electrons and fluorine narrow, due to annihilation from the o-Ps state in the free volume pores, and the parabolic contribution due to the annihilation of positrons with free electrons.

Figure 1 shows the concentration dependences of the PS parameters for the PTFE-MWCNT composites.

\section{Results And Discussion}


The maxima of $\{r\}_{-}\left\{\{m\}_{-}\{i\}\right\},\{r\}_{-}\left\{\{m\}_{-}\{b\}\right\}_{,}\{P\}_{-}\left\{\{r\}_{-}\left\{\{m\}_{-}\{b\}\right\}\right\}$ and the minimum $\{P\}_{-}\left\{\{r\}_{-}\left\{\{m\}_{-}\{i\}\right\}\right\}$ at a filler concentration of 10 wt.\% (Fig. 1) are caused by the compression of the carcasses of carbon atoms due to additional screening with increasing free electrons density in MWCNTs. This is due to the transferred charge from the charged layer $\Delta R$ on the inner surface of the free volume pores of the PTFE matrix (Tao S. J. J (1972)).

In our opinion, the main feature of this system is that the Fermi angle $\{\backslash \text { theta }\}_{-}\{F\}$ decreases monotonically over the entire concentration range, while the probability of annihilation of positrons with free electrons $\{P\}_{-}\left\{\{\backslash \text { theta }\}_{-}\{F\}\right\}$ increases in this case, reaches a maximum at a MWCNTs concentration of 10 wt.\% and reaches saturation at values typical for pure MWCNTs. A decrease in $\{1 \text { theta }\}_{-}\{F\}$ and an increase in $\{P\}_{-}\left\{\{\backslash \text { theta }\}_{-}\{F\}\right\}$ in the range from 5 to 10 wt.\% MWCNTs in a composite with changes in other PS parameters described above are due to the fact that not only charge is transferred, but also the effective mass of electrons changes greatly. It is not possible to separate these processes within the framework of the ACAR method; either the effective electrons masses or the free electrons densities obtained in the framework of other methods are needed, at least for several reference points.

Note again that the maxima $\{r\}_{-}\left\{\{\mathrm{m}\}_{-}\{b\}\right\}$ and $\{P\}_{-}\left\{\{r\}_{-}\left\{\{m\}_{-}\{b\}\right\}\right\}$ at 10 wt.\% MWCNTs are due to the high charge of Stone-Welsh defects in MWCNTs. The maximum $\{r\}_{-}\left\{\{\mathrm{m}\}_{-}\{i\}\right\}$ and the minimum $\{P\}_{-}\left\{\{r\}_{-}\left\{\{\mathrm{m}\}_{-}\{i\}\right\}\right\}$ at $10 \mathrm{wt}$.\% MWCNTs are due to the high free electrons concentration in MWCNTs; therefore, the free electrons density in the composite with $10 \mathrm{wt}$ \% MCNTs is higher than in the composite with 5 wt.\% MCNTs.

At high concentrations of MWCNTs in polymers (15-20 wt.\%), strong aggregation of nanotubes is observed (Garkusha O. M. (2010)); the polymer does not "wetting" the nanotubes. In this case, the volume of the interphase, at the interface of which the charge transfer occurs, greatly decreases; isolated bundles of nanotubes are pressed into the polymer matrix. Accordingly, in the concentration range of 15-20 wt.\% MWCNTs in the composite, the values of $\{\backslash \text { theta }\}_{-}\{F\}$ and $\{P\}_{-}\left\{\{\backslash \text { theta }\}_{-}\{F\}\right\}$ do not differ from the values characteristic of pure MWCNTs (Tsapko E. A. (2020)). Despite this, the conductive network formed by the nanotubes is preserved. All studied samples, except for pure PTFE, had finite electrical resistance.

Figure 2 Concentration dependence of absorption of electromagnetic radiation at a frequency of $2 \mathrm{GHz}$ in composites PTFE-MWCNT (Lihtorovich S. P. (2010))

It was found that the highest absorption of electromagnetic radiation at a frequency of $2 \mathrm{GHz}$ in PTFEMWCNT composites is observed in a sample with $10 \mathrm{wt} . \%$ MCNT.

Figure 3 shows the spectral dependences of the refractive index $n$ and the absorption coefficient $k$ at three different angles of incidence for a composite with 10 wt.\% MWCNTs.

For a PTFE composite with $10 \mathrm{wt}$ \% MWCNTs in the near ultraviolet region, a small peak is observed in the spectral dependence of the refractive index $n$. The absorption index $k$ increases monotonically over the entire investigated range of incident radiation frequencies. Such spectral dependences of the 
refractive indices $\mathrm{n}$ and absorption coefficient $k$ are characteristic of some allotropic forms of graphite (Ermolaev G. A. (2020)), where a small peak in the spectral dependence of the refractive index $n$ in the near-ultraviolet region (200-400 nm) corresponds to $s p^{2}$-hybridized states and a further monotonic increase in the refractive indices $n$ and absorption indices $k$ is due to transitions collectivized r-electrons of carbon. In pure PTFE, fundamentally different spectral dependences of the indices $n$ and coefficients $k$ are observed - low absorption and small changes (of the order of $10^{-1}$ ) over the entire investigated interval (Yang M. K. (2008)).

The dependences of the electronic structure and electromagnetic radiation absorption parameters on a frequency of $2 \mathrm{GHz}$ in the PTFE-MWCNT composites on the probability of annihilation of positrons with free electrons $\{P\}_{-}\left\{\{\backslash \text { theta }\}_{-}\{F\}\right\}$. are shown in Fig. 4.

The density of free electrons with a low effective mass in a sample with $5 \mathrm{wt} . \%$ MWCNTs is higher than in pure MWCNTs, because in this case, $\{r\}_{-}\left\{\{m\}_{-}\{b\}\right\}$ and $\{P\}_{-}\left\{\{r\}_{-}\left\{\{m\}_{-}\{b\}\right\}\right\}$ are significantly higher than in pure MWCNTs (Fig. 4), but this is not high enough to additionally screen the skeletons of carbon atoms, since $\{P\}_{-}\left\{\{r\}_{-}\left\{\{\mathrm{m}\}_{-}\{i\}\right\}\right\}$ of the composite is still higher, and $\{r\}_{-}\left\{\{\mathrm{m}\}_{-}\{i\}\right\}$ is lower than in pure MWCNTs (Fig. 4). Thus, in the concentration range from 5 to 10 wt.\% MWCNTs in the composite, the growth and densification of conducting chains continues (Garkusha O. M. (2010)).

The general trend of a decrease in the relative concentration $f_{r}$ of the free volume after the percolation threshold coincides with the trend described in (Ermolaev G. A. (2020), Yang M. K. (2008)). Average radius $R$ of free volume pores at 10 wt.\% MWCNT in the composite reaches its maximum value (Fig. 4).

We particularly note that the average of the free volume pores radius $R$ and the probability of annihilation of positrons $P_{R}$ in them are determined only by the parameters of the polymer matrix electronic structure, while the Fermi angle $\{\backslash \text { theta }\}_{-}\{$F $\}$and the probability of annihilation of positrons with free electrons $\{P\}_{-}\left\{\{\backslash \text { theta }\}_{-}\{F\}\right\}$ are determined only by the electronic structure of the MWCNT parameters. The rest of the PS parameters are determined by the electronic structure of both the polymer matrix and MWCNTS respectively. Because the skeleton electronic structure (parameters $\{r\}_{-}\left\{\{\mathrm{m}\}_{-}\{i\}\right\}$ and $\left.\{P\}_{-}\left\{\{r\}_{-}\left\{\{\mathrm{m}\}_{-}\{i\}\right\}\right\}\right)$ and defects electronic structure (parameters $\{r\}_{-}\left\{\{m\}_{-}\{b\}\right\}$ and $\left.\{P\}_{-}\left\{\{r\}_{-}\left\{\{m\}_{-}\{b\}\right\}\right\}\right)$ of the polymer matrix, at least outside the interphase, do not change, then the dynamics and of these parameters is due to the dynamics of the MWCNTs electronic structure.

In the PTFE-MWCNT composites, as can be seen from Fig. 4, in the sample with $10 \mathrm{wt} \%$ MWCNTs, the intensity of incident radiation at the exit from the sample decreases by a factor of 410 , i.e. almost $100 \%$ absorption is observed. Nanotubes do not emit absorbed energy in the form of photons.

In (Awad S. (2019)b), for the first time, an almost linear relationship was established between the average volume of nanopores of free volume $V_{f}$ in the $50 \%(\mathrm{Na}-\mathrm{CMC}) / 50 \%$ PAM + MWCNT composite and the energy that an excited electron can give to the phonon spectrum in 1 act of scattering on the "lattice" (Urbach energy $E_{U}$.). Most likely, in the PTFE-MWCNT composites, the numerical values of the $E_{U}$. versus 
$V_{f}$ dependence will be different. For approximate estimates, we take the value $E_{U}=0.58 \mathrm{eV}$ (Awad S. (2019)b), divide it by the Planck constant and see that for frequencies of electromagnetic radiation up to $1.40241 \cdot 10^{14} \mathrm{~Hz}$ (the wavelength of the incident radiation $\lambda=2.137659 \mu$ ), free electrons always have an alternative exit channel from the excited states - the phonon spectrum of the composite. High scattering of free electrons by phonons was also noted in (Xue G. (2016), Zhong J. (2019)) when studying the thermal and electrical conductivity of composites filled with reduced graphene oxide.

From the dependences of the absorption of electromagnetic radiation at a frequency of $2 \mathrm{GHz}$ on the electronic structure parameters of composites (Figs. 5, 6), it can be seen that at $5 \mathrm{wt} . \%$ MWCNTs, despite the high value of $\{\backslash \text { theta }\}_{-}\{F\}$ (high density and / or low effective mass of free electrons), the composite, like pure PTFE, is transparent to electromagnetic radiation. Consequently, it is not isolated MWCNTs that absorb electromagnetic radiation, but conductive circuits of MWCNTs, the process of formation and densification of which occurs in the concentration range from 5 to $10 \mathrm{wt}$ \% MWCNT in the composite. Therefore, the dependence of the absorption of electromagnetic radiation at a frequency of $2 \mathrm{GHz}$ on the average free volume pore radius $R$ has a complex nonlinear form (Fig. 5 (c)) and is determined by the formation and destruction of dense conducting circuits in the composite.

The electromagnetic radiation absorption at a frequency of $2 \mathrm{GHz}$ increases with an increase in the density of free electrons in MWCNTs (Fig. 6 (c, d)) in the presence of dense conducting chains in the composite, although such absorption is not observed in pure nanotubes. This effect is due to the fine structure of the density of states of carbon $\pi$-electrons in composites near the Fermi level. In MWCNTs, as in semimetals, a sufficiently high density of almost free $\pi$-electrons and, at the same time, an almost zero density of states at the Fermi level - there are almost no allowed free states above the Fermi level, and in processes of small excitations, almost free $\pi$-electrons are not participate. In composites with MWCNTs, as a result of charge flow from the matrix to MWCNTs (or vice versa, depending on the difference between the average layer charges $\Delta R$ on the inner surface of the pores of the matrix free volume and the Stone-Welsh vacancy), the chemical potential changes and immediately above it, allowed states appear in the density of states, which leads, as can be seen from the spectral dependence of the refractive indices $\mathrm{n}$ and absorption $k$ (Fig. 3), to an almost linear increase in absorption in the entire investigated wavelength range. Moreover, from our point of view, the same $100 \%$ absorption should be observed in the entire infrared range of radiation.

\section{Declarations}

On behalf of all authors, the corresponding author states that there is no conflict of interest.

\section{References}

E. A. Tsapko and I. Ye. Galstian, Positron Spectroscopy Study of Structural Defects and Electronic Properties of Carbon Nanotubes, Progress in Physics of Metals,. 21, 153-179 (2020).

https://doi.org/10.15407/ufm.21.02.153

Page $7 / 13$ 
M. M. Nishchenko, E. A. Tsapko, Yu. V. Lisunova, G. P. Prikhod'ko, and N. I. Danilenko, Electron-positron annihilation in carbon nanotubes, Inorg. Mater. Appl. Res. 2, 186-191 (2011). https://doi.org/10.1134/S2075113311020158.

S. P. Lihtorovich, M. M. Nishchenko, I. E. Galstyan, E. M. Rudenko, I. V. Korotash, O. I. Rzheshevskaya, G. P. Prihod'ko, and N. A. Gavrilyuk, Influence of nanotubes on the parameters of nanopores and radio absorption at a frequency of $2 \mathrm{~Hz}$ in fluoroplastic F4, Metallofiz. Noveishie Tekhnol., 32, No 4, p. 475 (2010) (in Russian)

S. Awad, S. El-Gamal, A. M. El Sayed, and E. E. Abdel-Hady, Physical properties of the organic polymeric blend (PVA/PAM) modified with MgO nanofillers, Polym Adv Technol.; 53, No 20, 1 (2019). https://doi.org/10.1177/0021998319840802

G. Xue, J. Zhong, Sh. Gao, and B. Wang, Correlation between the free volume and thermal conductivity of porous poly(vinyl alcohol)/reduced graphene oxide composites studied by positron spectroscopy, Carbon, 96, 871 (2016). https://doi.org/10.1016/j.carbon.2015.10.041

L. V. Poperenko, V. S. Stashchuk, I. A. Hajkevich, and V. A. Odarich, Diagnostics of surface with polarized light: Monograph, Vidavnicho-polyagrafichnij centr «Kiïvs'kij universitet», 336 (2007) (in Russian)

S. J. J. Tao, Positronium Annihilation in Molecular Substances, Chem. Phys., 56, No 11, 5499 (1972). https://doi.org/10.1063/1.1677067

O. M. Garkusha, S.M. Makhno, G.P. Prikhodko, Yu.I. Sementsov, and M.T. Cartel, Structural features and power of polymer nanocomposites at low concentrations of filler, Chem., Phys. and Techn. of the Surface, 1, No 1, 103-110 (2010). (in Ukrainian)

G. A. Ermolaev, A.P. Tsapenko, V. S. Volkov, A. S. Anisimov, Y. G. Gladush, and A. G. Nasibulin, Express determination of thickness and dielectric function of single-walled carbon nanotube films, Appl. Phys. Lett., 116, 231103 (2020). https://doi.org/10.1063/5.0012933

M. K. Yang, Optical properties of Teflon® AF amorphous fluoropolymers, Journal of Micro/Nanolithography, MEMS, and MOEMS, 7(3), 033010, (2008). https://doi.org/10.1117/1.2965541

S. Awad, S. El-Gamal, A. M. El Sayed, and E. E. Abdel-Hady, Characterization, optical, and nanoscale free volume properties of Na-CMC/PAM/CNT nanocomposites, Polym Adv Technol., 31, 1-12, (2019). https://doi.org/10.1002/pat.4753

J. Zhong, Y. Ding, F. Gao, J. Wen, J. Zhou, W. Zheng, L. Shen, Ch. Fu, and B. Wang, Free volume correlation with electrical conductivity of polycarbonate/reduced graphene oxide nanocomposites studied by positron annihilation lifetime spectroscopy, Inc. J. Appl. Polym. Sci., 136, 48207 (2019). https://doi.org/10.1039/D0RA05872F 
Figures

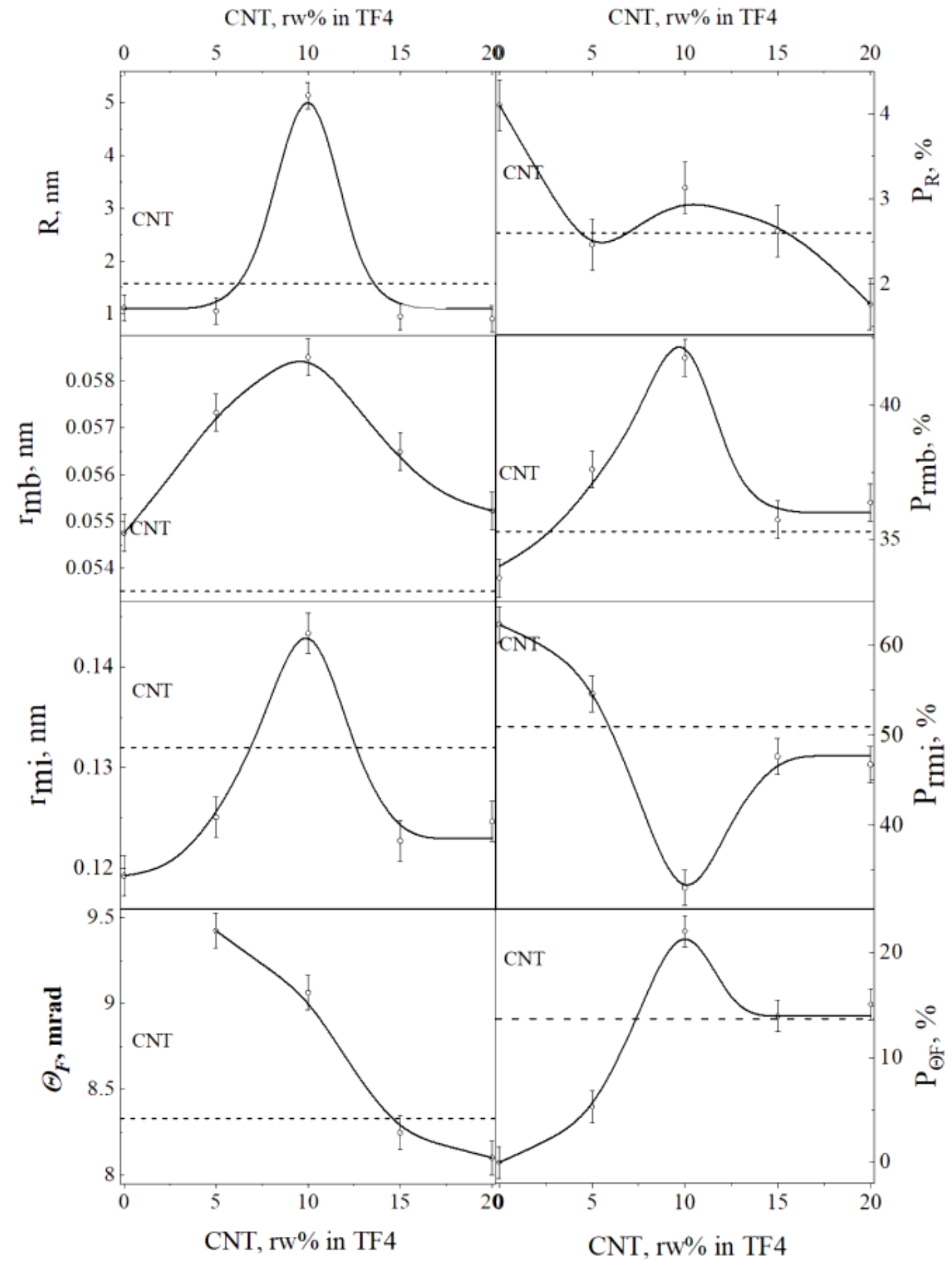

Figure 1

Concentration dependences of PS parameters in PTFE-MWCNT composites 


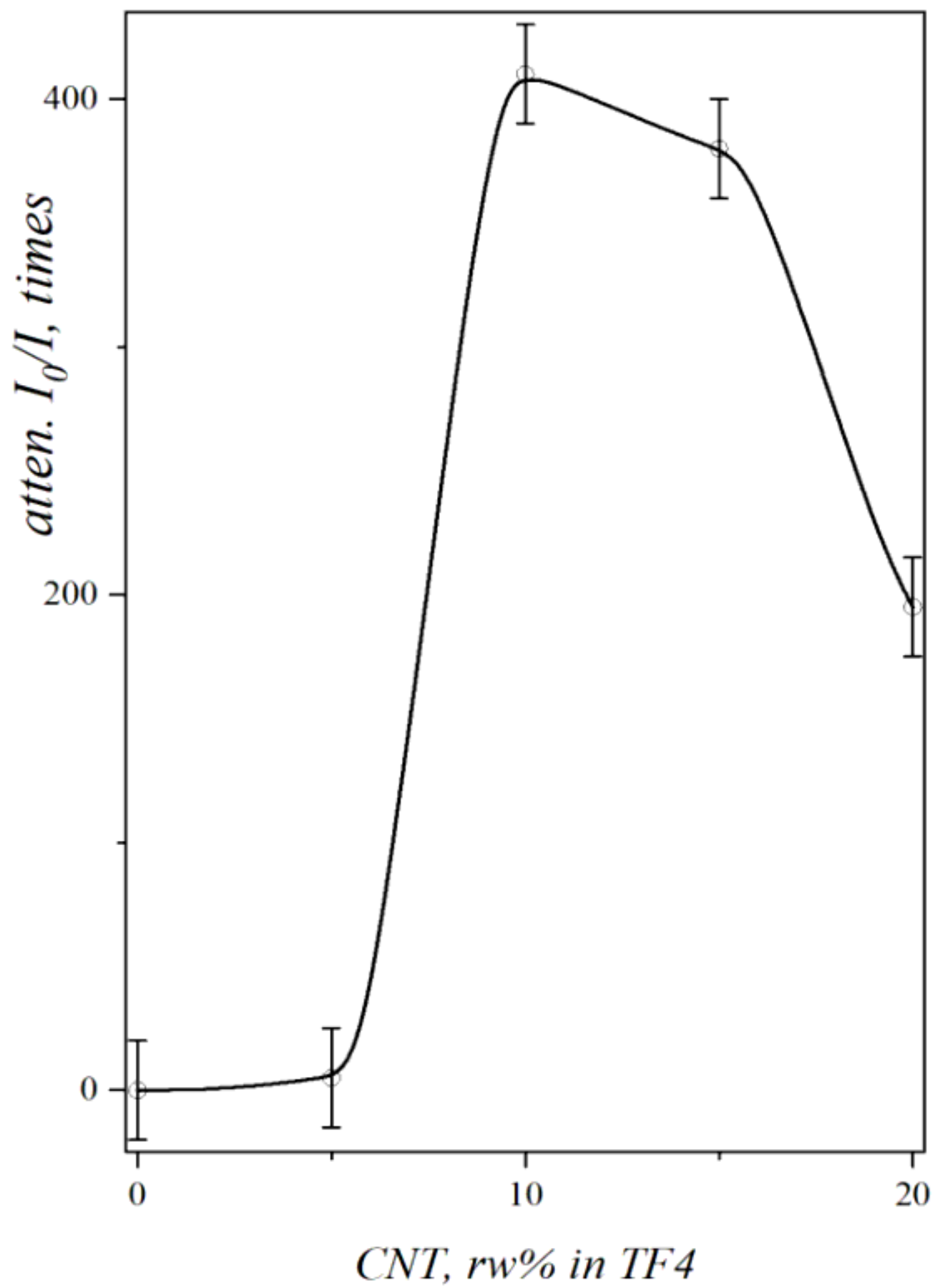

Figure 2

Concentration dependence of absorption of electromagnetic radiation at a frequency of $2 \mathrm{GHz}$ in composites PTFE-MWCNT (Lihtorovich S. P. (2010))

Figure 3 
Spectral dependences of refractive index $n$ and absorption coefficient $k$ at three different angles of incidence for a composite with 10 wt.\% MWCNTs

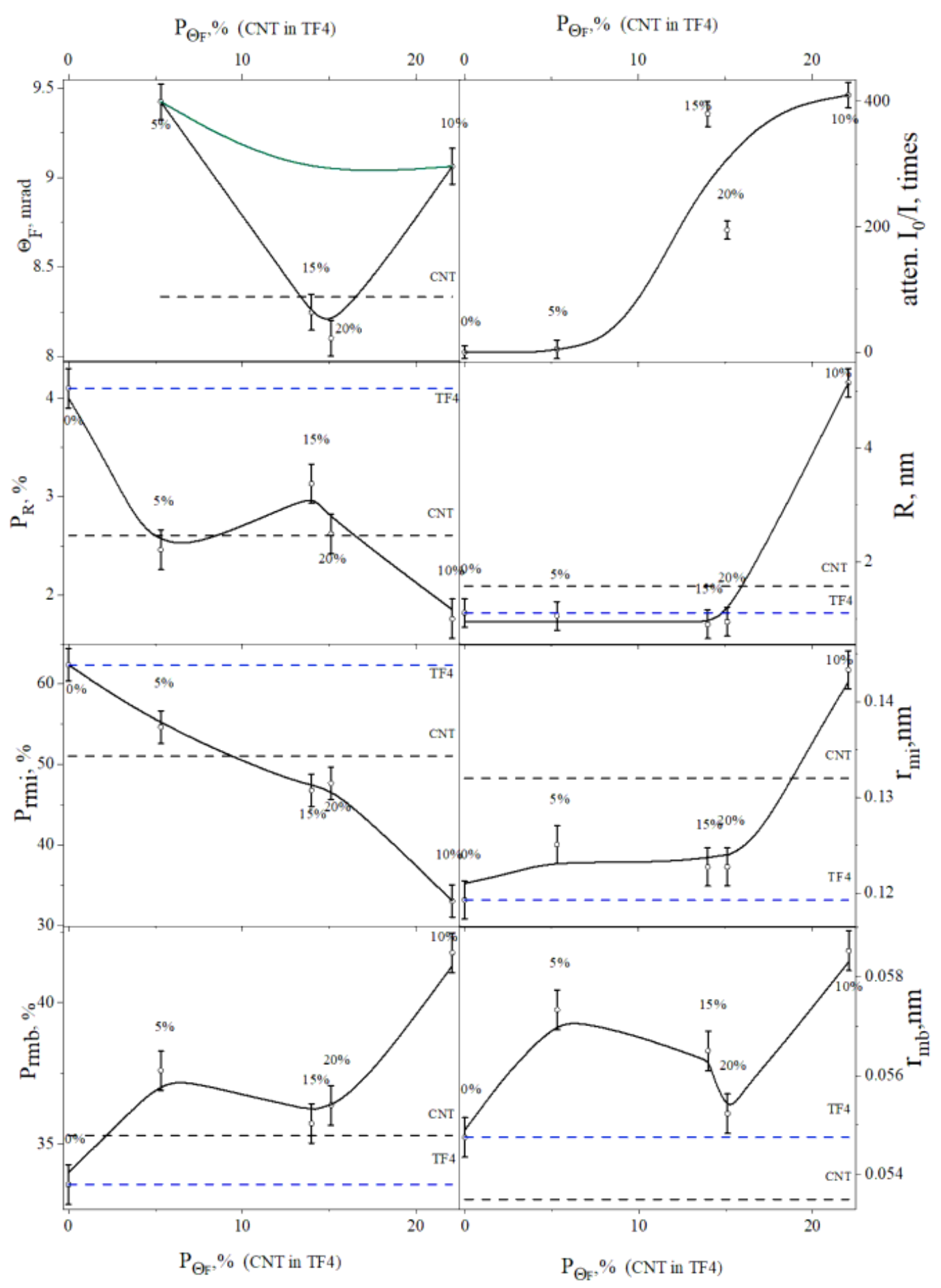

Figure 4

Dependences of the electronic structure parameters and the electromagnetic radiation absorption at a frequency of $2 \mathrm{GHz}$ in the PTFE-MWCNT composites on the probability of annihilation of positrons with 
free electrons $P_{\theta F}$

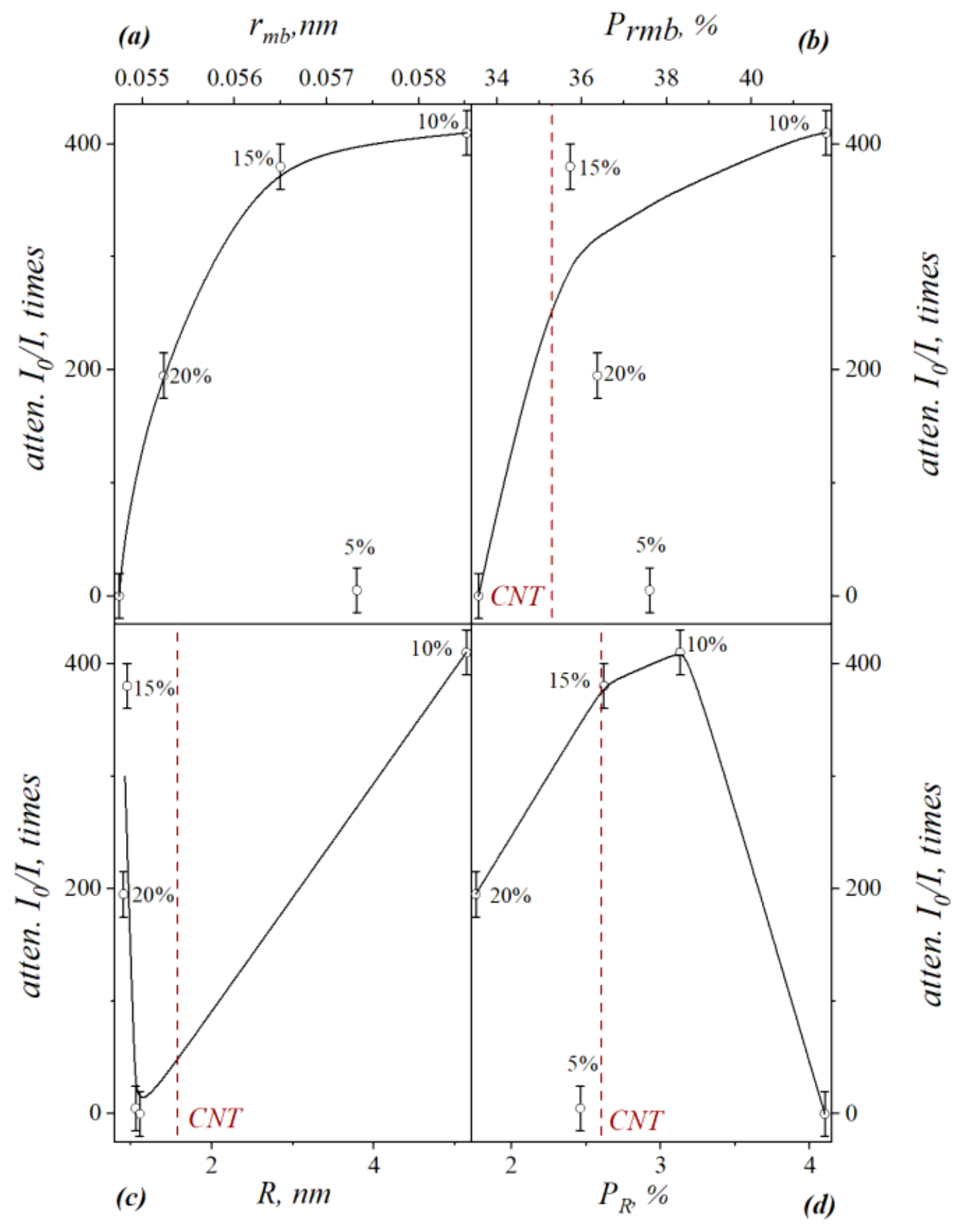

Figure 5

Dependences of the electromagnetic radiation absorption at a frequency of $2 \mathrm{GHz}$ in the PTFE-MWCNT composites on the electronic structure parameters $\mathrm{r}_{\mathrm{mb}}, \mathrm{P}_{\mathrm{rmb}}, R$ and $P_{R}$ 


\section{Figure 6}

Dependences of the absorption of electromagnetic radiation at a frequency of $2 \mathrm{GHz}$ in the PTFEMWCNT composites on the parameters of the electronic structure $r_{\mathrm{mi}}, \mathrm{P}_{\mathrm{rmi}}, \theta_{F}$ and $P_{\theta F}$. 